\title{
The role of macrophages in the resolution of inflammation
}

\author{
Satoshi Watanabe,,$^{1,2}$ Michael Alexander, ${ }^{1}$ Alexander V. Misharin, ${ }^{1}$ and G.R. Scott Budinger ${ }^{1}$ \\ 'Division of Pulmonary and Critical Care Medicine, Northwestern University, Feinberg School of Medicine, Chicago, Illinois, USA. Department of Respiratory Medicine, Kanazawa University Graduate School \\ of Medical Sciences, Kanazawa, Ishikawa, Japan.
}

\begin{abstract}
Macrophages are tissue-resident or infiltrated immune cells critical for innate immunity, normal tissue development, homeostasis, and repair of damaged tissue. Macrophage function is a sum of their ontogeny, the local environment in which they reside, and the type of injuries or pathogen to which they are exposed. In this Review, we discuss the role of macrophages in the restoration of tissue function after injury, highlighting important questions about how they respond to and modify the local microenvironment to restore homeostasis.
\end{abstract}

\section{Introduction}

Macrophages are innate immune cells present in every tissue and necessary for homeostasis. Macrophages sense and respond to pathogens and other environmental challenges and participate in tissue repair after injury. Work from many groups in the last decade reveals macrophages as remarkably plastic cells that are epigenetically programmed in response to signals originating from the tissue environment $(1,2)$. Macrophages integrate endocrine or paracrine signals with signals originating from phagocytosed cells, microvesicles, and molecules in the extracellular matrix. In addition, macrophages can interact directly with surface receptors on other tissue-resident cell populations, immune cells recruited during injury, and extracellular proteins. As a result, macrophages play diverse roles in development, the acute response to infection and tissue injury, and tissue repair. Because macrophages play tissue- and disease stage-specific roles, therapies that target them might be expected to have fewer of the off-target effects that limit the use of less selective therapies. Achieving this goal will require more precise molecular endotyping and targeting of macrophage subpopulations over the course of tissue injury and repair. Here we describe recent advances in our understanding of the origin, subtype, and phenotype of tissue macrophages during homeostasis and repair.

\section{Macrophages in homeostasis}

Developmental origins of tissue macrophages. In 1968, based on labeling studies after whole-body irradiation, van Furth and colleagues proposed that bone marrow-derived circulating monocytes are the source of tissue macrophages (3). This paradigm was overturned within the last decade when several independent groups reported the results of genetic lineage tracing studies in mice (4-10). They found that in many tissues, macrophages originate from precursor cells derived from the yolk sac or fetal liver and differentiate into macrophages as part of prenatal or antenatal development. These

Conflict of interest: The authors have declared that no conflict of interest exists. Copyright: (c) 2019, American Society for Clinical Investigation.

Reference information: J Clin Invest. 2019;129(7):2619-2628.

https://doi.org/10.1172/JCI124615. "tissue-resident macrophages" can be very long-lived (months to years in the brain, liver, lung, and skin) and self-renewing, maintaining their homeostatic pool without a contribution from circulating monocytes (11-13). In other tissues, tissue-resident macrophage populations are replaced by monocyte-derived cells over different time scales. For example, in the intestine, locally maintained tissue-resident macrophages coexist with monocytederived populations with relatively short half-life, which have distinct roles in gut homeostasis and intestinal physiology (14-16).

Advances in flow cytometry, lineage tracing systems, and insights from single-cell transcriptomics have dramatically improved our ability to identify distinct macrophage populations (17). For example, a recent study identified at least two unique tissue-resident interstitial macrophages in the steady-state lung that could be distinguished by unique transcriptional profiles and spatially localized to the interstitium of the bronchovascular bundles, but not alveolar walls (18). Indeed, most tissues are now recognized to contain multiple macrophage populations localized to distinct microanatomical domains (18-20). Each of these populations differs in its ontogeny, rate of replacement by monocytederived cells, and capacity for self-renewal, and each is likely to play a specialized role in tissue homeostasis, injury, and repair (Figure 1 and refs. 13, 21). The application of single-cell transcriptomics and high-throughput spatial transcriptomics in mice and humans combined with advanced lineage tracing studies in mice will allow a more complete understanding of the spectrum of macrophage phenotypes in different microdomains within healthy and diseased tissues. These same technologies can be used to generate and test hypotheses with respect to the molecular mechanisms by which macrophages contribute to tissue injury and repair and how they can be targeted for therapy (19).

Epigenetic control of macrophage differentiation is tissue-specific. Relative to dendritic cells, tissue macrophages poorly present antigens to other immune cells and fail to migrate to regional lymph nodes (22). Transcriptomic profiling of four tissue-resident macrophage populations by the ImmGen consortium revealed that macrophages from different tissues - brain microglia, splenic red pulp macrophages, large peritoneal macrophages, and Kupffer cells in the 


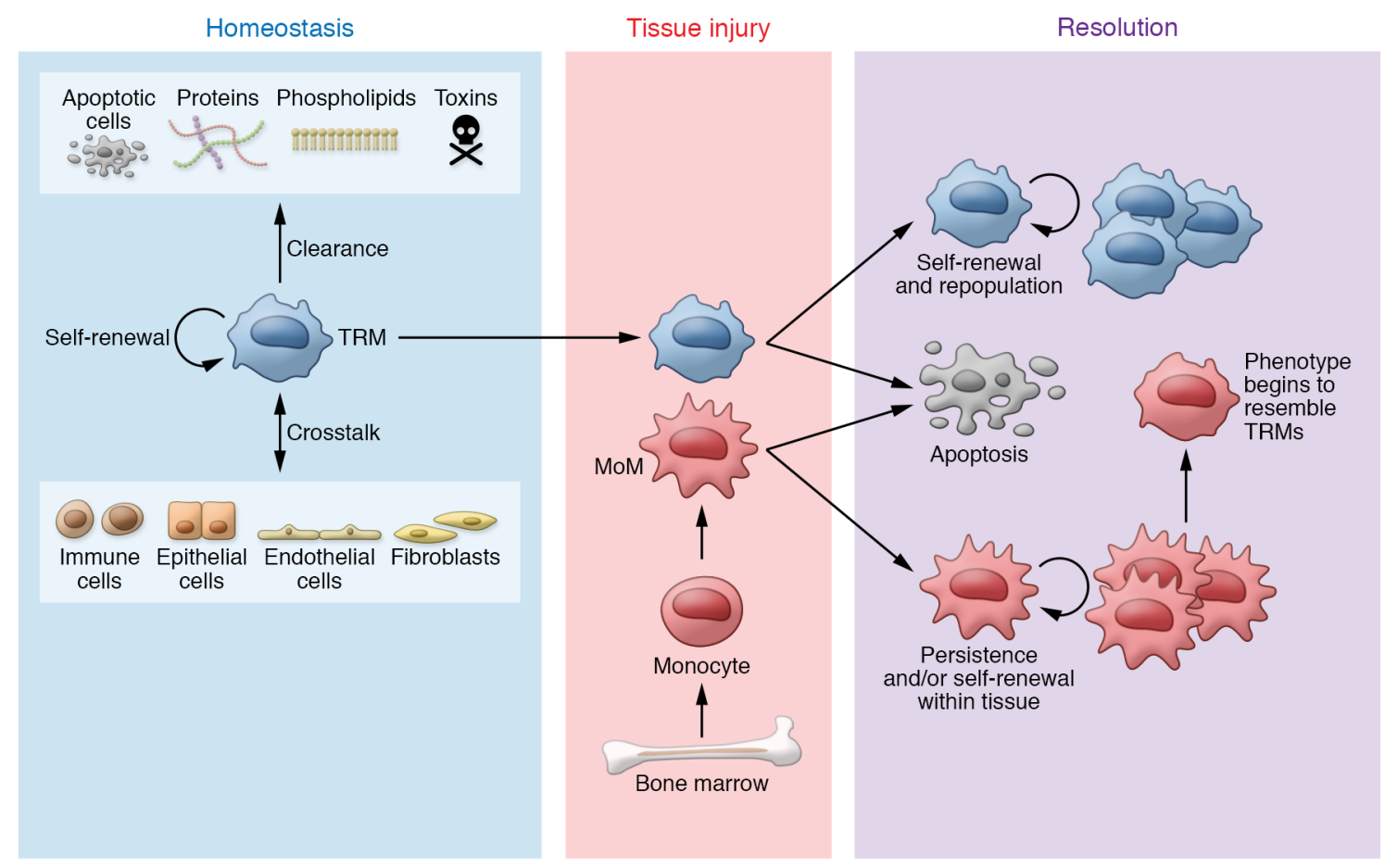

Figure 1. Tissue-resident macrophages and monocyte-derived macrophages play distinct roles in tissue injury and repair. Tissue-resident macrophages (TRMs) originate from the yolk sac and fetal liver during development and persist in many tissues via self-renewal. During homeostasis (left panel), TRMs clear apoptotic cells, proteins, and phospholipids and either clear or respond to toxins, particulates, and pathogens within the local microenvironment. Many TRMs are capable of maintaining themselves by local proliferation without the contribution of monocyte-derived macrophages (MoMs). TRMs produce a variety of factors that stimulate the activation, proliferation, and differentiation of immune cells, epithelial cells, endothelial cells, fibroblasts, and stem cells that facilitate tissue homeostasis. In response to tissue injury (middle panel), bone marrow-derived monocytes are recruited to the injured tissue, where they differentiate into MoMs. During injury, TRMs and MoMs play distinct roles; usually MoMs exhibit a more robust inflammatory response. During the resolution of injury (right panel), TRMs may die or expand through self-renewal and repopulate the niche. MoMs either undergo apoptosis or persist, sometimes gaining the capacity for self-renewal. Over time, the phenotypes of TRMs and MoMs become increasingly similar. Arrows indicate interactions with other cell types.

liver - had more differences in their transcriptional program than similarities (23). In contrast, dendritic cells recovered from a variety of tissues had more similar transcriptomes. These findings suggested that tissue-resident macrophages were uniquely defined by factors originating from their microenvironment. In seminal studies, two groups of investigators used transcriptional and epigenomic profiling of tissue-resident macrophage populations to provide insights into the molecular events that allow monocytes to differentiate along widely divergent paths in a tissue-specific context $(2,24)$. They found that macrophage populations were distinguished from other myeloid cells and each other at the epigenetic level as measured by histone modifications in both promoters and enhancers. These epigenetic modifications determined tissue macrophage transcriptomic identity. Enhancers are regions distal to the transcriptional start site of genes marked by the corresponding histone marks (H3K4me1 for poised enhancers or H3K27ac for active enhancers). Most macrophage-specific enhancers contain binding domains for the pioneering transcription factor PU.1. Tissue-specific macrophages are further distinguished by enrichment in tissue-specific transcription factor binding domains, for example GATA-6 in peritoneal macrophages, MEF2 in microglia, LXR $\alpha$ in Kupffer cells and spleen macrophages, and PPAR $\gamma$ in alveolar and spleen macrophages. Importantly, these epigenetic enhancer landscapes are similar both in naive mice (embryonically derived macrophages) and in macrophages derived from monocytes after total-body irradiation and congenic bone marrow reconstitution, suggesting that fully developed tissues retain the ability to epigenetically program monocytes into tissue-resident macrophages. Furthermore, these epigenetic changes are reversible, as mature macrophages adoptively transferred from one tissue to another take on the phenotype of the recipient tissue macrophages $(2,25,26)$. Together these findings support a model whereby the tissue microenvironment continuously provides signals that reversibly induce macrophage differentiation in a tissue-specific context through the hierarchical recruitment of transcription factors that alter the epigenetic landscape of the cell. Given the dramatic changes in environmental signals associated with tissue injury, these results suggest important plasticity in macrophage responses as the microenvironment changes.

Macrophages during tissue injury. For almost a century, investigators have focused on the role of monocytes and macrophages in the acute response to tissue injury, where they are known to produce cytotoxic and proinflammatory mediators, clear invading microorganisms, remove apoptotic and damaged cells, and promote tumor progression $(27,28)$. Chemokine receptor 2 (CCR2) is required for the release of monocytes from the bone marrow and the recruitment of monocytes to tissues during injury. Mice deficient in CCR2 are therefore monocytopenic and fail to recruit monocytes and monocyte-derived macrophages to tissues during 
A
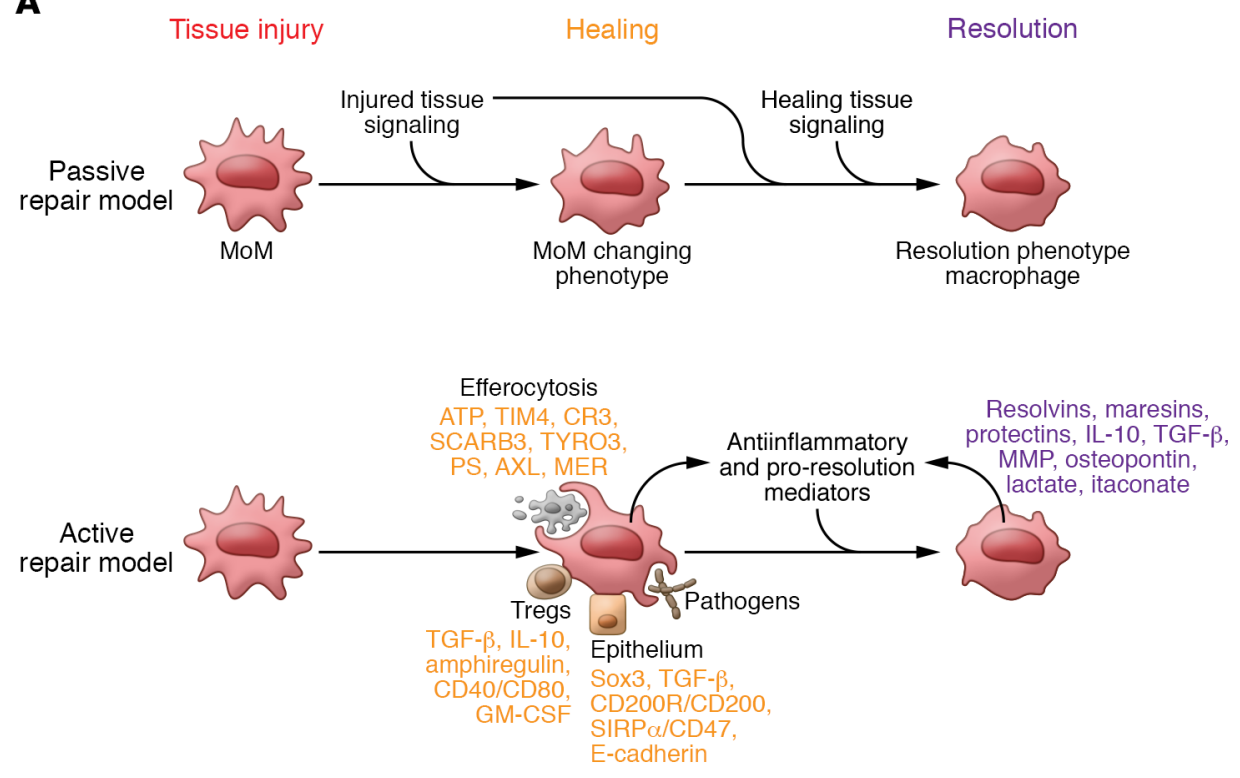

B

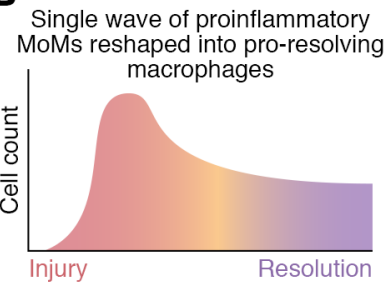

Proinflammatory MoMs disappear and replaced by recruited

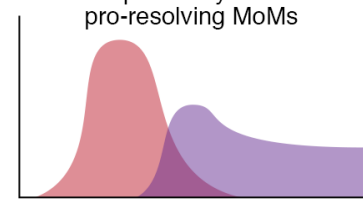

MoMs of varying function recruited through injury and resolution

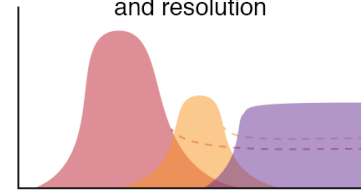

Figure 2. Role and kinetics of macrophages during tissue injury and repair. (A) Monocytes are recruited to the tissue during injury, where they differentiate into macrophages in response to cues provided by the injured microenvironment. We propose two models to understand the distinct roles of monocytes in promoting tissue injury and tissue repair during injury resolution, which are not mutually exclusive. In the passive repair model (top panel), tissue regeneration restores signals that promote macrophage differentiation into cells that increasingly resemble tissue-resident macrophages. As the homeostatic function of macrophages is restored, tissue repair is accelerated, creating a feed-forward loop that restores homeostasis. In the active repair model (bottom panel), monocyte-derived macrophages respond to cues in their microenvironment and express or secrete factors that drive tissue repair. Interactions include the uptake of apoptotic cells (often neutrophils), regulatory T cells, pathogens, and epithelial cells. These monocyte-derived macrophages might promote the resolution of inflammation through secretion of antiinflammatory and pro-repair mediators including metabolic intermediates, pro-resolution lipid mediators, antiinflammatory cytokines, and matrix remodeling proteins. (B) The kinetics of monocyte-derived macrophage recruitment to tissues is a subject of active investigation. A single wave of monocytes may enter during injury and be progressively reshaped into pro-resolving macrophages in response to cues within the local microenvironment (top panel). Alternatively, distinct waves of monocyte-derived macrophages might be involved in tissue injury (red) and tissue repair (purple) (middle panel), or monocytes with varying functions might be continuously recruited over the course of tissue injury and repair (bottom panel).

injury. CCR2-deficient mice or genetic or pharmacologic deletion of CCR2-positive monocytes have been widely used to demonstrate the importance of monocytes and/or monocyte-derived cells in the development of tissue injury and the clearance of invading pathogens (29-32). More recently, investigators have used selective deletion strategies to specifically demonstrate a contribution of monocyte-derived alveolar macrophages to tissue injury and fibrosis, in some cases excluding a role for tissue-resident macrophages (reviewed below). Comparisons of monocyte-derived and tissue-resident macrophages colocalized in the injured tissue using bulk or single-cell RNA-Seq reveal distinct transcriptional profiles during injury $(29,33)$. In general, both tissue-resident and monocyte-derived macrophages demonstrate qualitatively similar changes in gene expression in response to injury, but these responses are more robust in monocyte-derived cells (9) and disproportionately affect physiologic measures of injury.

Collectively, these findings highlight the importance of macrophage ontogeny during acute injury with important consequences for the interpretation of both published and prospective studies. Specifically, experimental strategies that target genes necessary for monocyte-to-tissue macrophage differentiation have to be interpreted with caution. For example, monocyte-to-alveolar macrophage differentiation has been reported to require several genes, including Torc1, Pparg, and Tgfb1 (34-38). Deletion of these genes in monocytes or differentiating macrophages (for example with a LysM-Cre or CD11c-Cre system) will therefore prevent or slow accumulation of monocyte-derived macrophages in the tissues upon the injury (39). As a result, it is impossible to distinguish effects on tissue injury or repair secondary to depletion of monocyte-derived cells from those related to the specific functions of the targeted gene unless the study is combined with lineage tagging to distinguish monocyte-derived and tissue-resident cells. Similar concerns might affect tissue-specific transcription factors important for macrophage differentiation in other tissues, for example MEF2C in microglia, LXR $\alpha$ in Kupffer cells and splenic macrophages, PPAR $\gamma$ in splenic red pulp and lung, GATA- 6 in peritoneal macrophages, and RUNX3 in intestinal macrophages (2).

Breaking out of the M1/M2 box. In cultured bone marrowderived macrophages, the administration of LPS and IFN- $\gamma$ induces genes encoding proinflammatory cytokines, including TNF- $\alpha$ and IL-6. In contrast, the administration of IL- 4 and IL-13 results in the expression of antiinflammatory cytokines, including IL-10, TGF- $\beta$, 


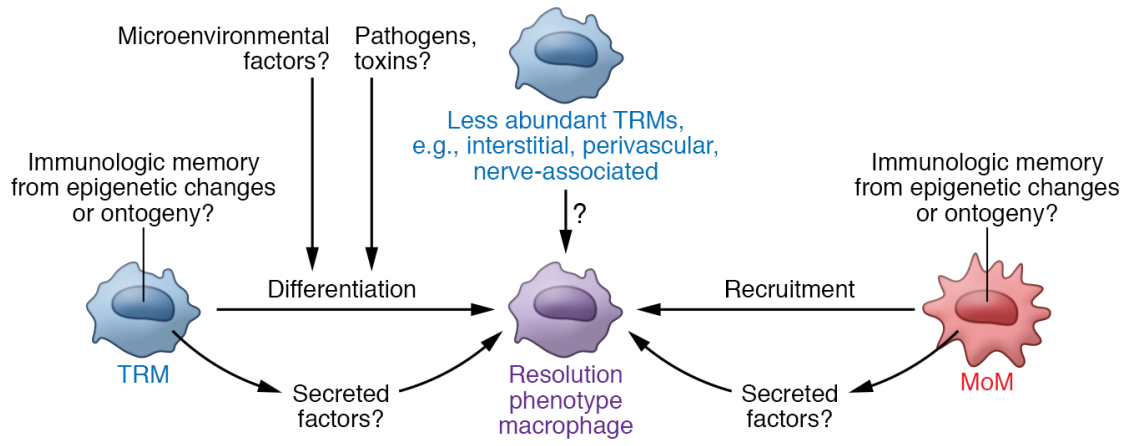

Figure 3. Major questions remaining regarding the role of macrophages in tissue injury and repair.

resident tissue microenvironment in which the injury occurs, and the ontogeny of the macrophages. In addition, the role of any given macrophage population in tissue injury and repair can change dramatically with time, which might explain occasionally divergent results in the same model system. The molecular events that orchestrate the changing roles for different macrophage populations over the course of tissue injury and repair are beginning to be understood. Some of these common mechanisms are discussed below.

Macrophages as active or passive participants in tissue repair. During tissue injury,

and the cytosolic enzyme arginase-1. As initial analyses included only a handful of nonoverlapping markers, a conceptual framework evolved that limited macrophages to an "M1" or "M2" phenotype in an in vivo context, analogous to the Th1/Th2 concept that was dominating $\mathrm{T}$ cell biology at that time $(40,41)$.

While the framework of M1/M2 polarization has provided a useful system to study macrophages in vitro, it has hindered our understanding of macrophage plasticity in vivo, as classical M1 and M2 polarization is unlikely to occur in a tissue context. This was definitively shown by Xue et al., who found that the M1/M2 paradigm failed to describe the transcriptome of human monocytederived and alveolar macrophages stimulated with LPS/IFN- $\gamma$ or IL-4/IL-13 in the presence of factors known to be present in different tissue or disease microenvironments (42). These macrophage responses can be remarkably selective. For example, Avraham et al. used single-cell RNA-Seq to show that peritoneal macrophages in the same microenvironment respond differently to salmonella strains that differ by a single gene (43). The M1/M2 paradigm can be particularly misleading during acute injury, when tissue-resident and monocyte-derived macrophages coexist in the diseased microenvironment. Specifically, many tissue-resident macrophage populations express higher levels of "M2" markers when compared with maturing monocyte-derived macrophages $(2,13,33)$. As a result, in the absence of a lineage marker, the simultaneous presence of immature monocyte-derived and mature tissue-resident macrophages will result in apparent "M1" polarization of the mixed populations (on average). During repair, monocyte-derived macrophages increasingly mature and resemble tissue-resident macrophages, a process that can take weeks $(2,13,44)$. As a result, in the absence of a lineage mark, the mixed population will appear to "switch" to an M2 phenotype. These limitations likely explain emerging literature in which bulk RNA-Seq of flow cytometry-sorted macrophages and single-cell RNA-Seq data collected during tissue injury and repair in mice and humans reveal macrophage phenotypes that are inconsistent with the M1/M2 paradigm $(13,45)$. We join other authors who suggest strictly limiting the use of M1/M2 polarization to the well-defined in vitro conditions in which it was described $(46,47)$.

\section{Role of macrophages in tissue repair}

Macrophages have frequently been reported to play divergent roles in tissue injury and tissue repair (Figure 1). A better understanding of these roles might be obtained by considering unique factors associated with the environmental stimulus that induces the injury, the pathogens, infected cells, and cells dying from necroptosis or pyroptosis release pathogen- or damage-associated molecular patterns (PAMPs or DAMPS), which activate inflammatory signaling pathways in macrophages and other resident cell populations that recruit neutrophils, monocytes, and other inflammatory cells to the tissue. Once the acute injury has been controlled, macrophages play a role in suppressing inflammation and initiating wound repair by clearing debris and producing growth factors and mediators that provide trophic support to the tissue in which they reside (48). We suggest two nonexclusive pathways by which tissue macrophages might contribute to repair (Figure 2). The first process, which we refer to as "passive macrophage repair," involves the progressive differentiation of monocytederived macrophages in response to a growing number of "normal" signals originating from the regenerating tissue microenvironment. As this process of differentiation occurs, the macrophages take on phenotype and function increasingly similar to those of homeostatic tissue-resident macrophages. The result is a positive-feedback loop in which an increasing normalization of the tissue microenvironment drives a progressively more homeostatic role for macrophages, which in turn promote tissue repair. In this model, monocyte-derived macrophages may develop a capacity for self-renewal and persist in the tissue after resolution, perhaps through downregulation of the transcription factor MAFB $(38,45)$. Alternatively, monocyte-derived macrophages might die by apoptosis, allowing the restoration of tissue-resident macrophages through proliferation and migration, as was shown in microglia using an elegant fate-mapping system (49).

The second process, which we call "active macrophage repair," involves activation of specific transcriptional programs in macrophages in response to factors uniquely present in the injured tissue microenvironment. The best studied of these mechanisms involves macrophage efferocytosis of apoptotic neutrophils recruited to the tissue during injury (37). During efferocytosis, the externalization of intracellular phospholipids, including phosphatidylserine, and intracellular molecules, including ATP, on apoptotic cells induces the absorption of opsonins such as Mfge8 and AnxA1 onto the apoptotic cells' surface $(50,51)$, and induces binding of protein $S$ and GAS6 to phosphatidylserine. Protein S and GAS6 serve as ligands for the TAM receptor tyrosine kinase family (TAM is an acronym derived from the first letter of its three constituents: Tyro3, Axl, and Mer), whose activation is facilitated by TIM4 and bridging molecules including DEL-1 (52-54). Binding to the TAM receptors activates downstream transcription factors, including $\operatorname{LXR} \alpha, \operatorname{LXR} \beta$, and PPAR $\gamma$ (encoded by NR1H3, NR1H2, and PPARG, respectively), 
to attenuate inflammatory signaling pathways in macrophages. Surveys of tissue macrophages reveal that the receptors, pathways, and transcriptional responses induced by the uptake of apoptotic cells differ in different tissues, and even within different macrophage populations within the same tissue (51). Despite these differences, the uptake of apoptotic cells almost always reduces the expression of proinflammatory cytokines and chemokines from macrophages. In addition to efferocytosis, other factors in the injured tissue microenvironment can activate antiinflammatory signaling pathways in macrophages. For example, regulatory $\mathrm{T}$ cells that expand in the injured tissue can release amphiregulin, TGF- $\beta$, and IL-10, or directly interact with macrophages via ligand/surface interactions induced by CD40/CD80 (55). Microvesicles originating from macrophages or other recovering cell populations have been reported to carry signaling molecules including SOCS2 or signaling microRNAs that induce reparative phenotypes (56-60).

Mechanisms of macrophage-mediated tissue repair. Understanding how macrophages communicate with resident cell populations to promote tissue repair represents an active area of investigation. In the passive model, this process occurs through restoration of tissuespecific homeostatic macrophage functions. These include upregulation of molecules that interact with the epithelium to promote homeostasis. Examples include the receptor/ligand pairs CD200/ CD200R, signal regulatory protein $\alpha(\operatorname{SIRP} \alpha) / C D 47$, and CSF2R/ GM-CSF and immune/epithelial E-cadherin interactions (61-64). In the active model, macrophages secrete factors that actively promote tissue repair. These include antiinflammatory molecules (IL-10 and TGF- $\beta$ ), growth factors (VEGF, PDGFA), matrix metalloproteinases (MMP-8, -10, -28), and osteopontin (65-67). Macrophages are also thought to be the major source of resolvins, protectins, and maresins, long-chain fatty acid-derived lipid mediators shown to drive the resolution of tissue injury in a wide variety of pathologies (68). Study of these molecules has been limited by their short half-life, their susceptibility to degradation during tissue processing, the requirement for mass spectroscopy for their detection, and the lack of a known specific receptor on target cell populations (68). The generation of these lipid mediators is consistent with an important role for macrophage metabolism in regulating tissue repair after injury $(69,70)$. For example, time series transcriptional data from cultured macrophages during efferocytosis identified a key role for solute transporters in the sensing, sampling, and ingestion of apoptotic material (71). These changes resulted in a switch to a glycolytic phenotype with the extracellular release of lactate, which served a signaling function to inhibit inflammatory responses. In addition, investigators found that activated macrophages produce an endogenous metabolite, itaconate, resulting in the attenuation of inflammation (72).

Macrophages can also contribute to chronic organ dysfunction, perhaps through abnormal activation of repair processes. For example, cell-autonomous activation of mTOR signaling in macrophages induces a systemic granulomatous disease with features suggestive of sarcoidosis in multiple tissues (73). While both resident and recruited macrophages are important for the clearance of pathogens in multiple tissues, some pathogens might hijack macrophage repair pathways to persist (74). For example, investigators recently found that cadherin-containing junctional complexes between macrophages in a zebrafish model of mycobacterial infection allowed the mycobacteria to evade immune clearance (75).
Time and ontogeny are important determinants of macrophage function during repair. Understanding the role of macrophages in tissue repair experimentally requires consideration of the tissue, the type and severity of injury, the duration of time after injury, and the ontogeny of macrophages, creating challenges for investigators. Some examples are illustrative. In the lung, the deletion of monocyte-derived macrophages ameliorates fibrosis during bleomycin-induced lung injury $(13,76)$, but the depletion of both tissue-resident and monocyte-derived macrophages late in fibrosis delays resolution (76). In contrast, Madsen and Bugge reported that monocyte-derived alveolar macrophages were dispensable for the development of fibrosis, but were necessary for collagen breakdown in the intact skin $(77,78)$. In the lung, we found that monocytederived and tissue-resident alveolar macrophages persist after the resolution of influenza A or bleomycin injury, whereupon they become transcriptionally similar to tissue-resident alveolar macrophages (13). These findings are consistent with earlier studies of tissue-resident macrophage depletion with clodronate or radiation $(2,79)$. It is not known, however, whether monocyte-derived macrophages are recruited to the site of injury as a single wave of cells that differentiate into a reparative phenotype as tissue injury resolves, or whether monocytes newly recruited during resolution serve specific repair functions (a distinction that may also depend on the injurious stimulus) (Figure 2B). Alternatively, tissue-resident macrophages may disproportionately contribute to tissue repair. Finally, there is evidence to suggest that epigenetic changes in macrophages might be important in conferring innate immunologic memory $(80,81)$. Advances in lineage tracing methods and inducible deletion strategies combined with single-cell transcriptomic approaches to identify heterogeneity should provide answers to these questions.

Role of macrophages during aging. As age is the most important risk factor for many of the chronic disorders associated with macrophage dysfunction, a better understanding of age-related changes in macrophages will be important to understand their role in tissue repair (82). Several groups of investigators have observed impaired macrophage transcription and function in normal aging. These include reduced phagocytosis, impaired polarization in vitro, a loss of wound healing response, and a reduced response to Toll-like receptor activation (83). Investigators have used diverse models of injury and repair to show that aged macrophages drive degenerative phenotypes in the skin, peripheral nervous system, and vasculature (83-88). Microglial inflammatory activation and impaired phagocytosis in aged microglia have been implicated in the development of Alzheimer's disease (89). Low-grade activation of the NLRP3 inflammasome in adipose tissue macrophages impairs their ability to clear lipids in response to adrenergic stimulation during aging, contributing to age-related adiposity (90). Several questions remain to be explored. For example, are changes in macrophage function with aging cell-autonomous, or are agerelated changes in macrophage function driven by the loss or gain of signals from the microenvironment? Are tissues that harbor long-lived macrophage populations (for example, the brain, liver, skin, and lung) more or less susceptible to age-related diseases when compared with tissues in which macrophages are continuously replenished by bone marrow-derived cells? Do alterations in macrophage ontogeny drive differential responses in young compared with aged tissues? 


\section{Tissue-specific macrophage contributions to repair}

Any discussion of the role of macrophages in tissue repair across organs is necessarily incomplete, but some examples highlight consistent themes.

Heart. Investigators have identified four distinct populations of tissue-resident macrophages in the heart, which have been shown to play divergent roles in tissue injury and repair $(7,20)$. Resident CCR2-negative macrophages promote coronary development and cardiac regeneration and facilitate electrical conduction within the atrioventricular node $(91,92)$. The depletion of CCR2-positive (monocyte-derived) macrophages improved outcomes following myocardial infarction $(93,94)$, while depletion of tissue-resident populations after infarction led to impaired cardiac function and adverse remodeling primarily within the peri-infarct zone (20). Interactions between the macrophage scavenger receptor MER and apoptotic cells through the opsonin MFGE8 improve cardiac repair after myocardial infarction (95). Metabolic changes in cardiac macrophages induced via MERTK likely support their role in resolution. While further studies with more precise lineage tracing and deletion approaches are needed, these findings support the hypothesis that tissue-resident macrophages and recruited monocyte-derived macrophages play distinct roles in cardiac injury and repair.

Lung. Alveolar macrophages are long-lived tissue-resident macrophages localized to the alveolar space, where they play an important role in maintaining homeostasis and host defense in the lung $(96,97)$. Monocyte-derived alveolar macrophages recruited during injury contribute to the development of fibrosis in response to bleomycin, while tissue-resident alveolar macrophages do not $(13,98)$. Bulk RNA-Seq of flow-sorted monocyte-derived and tissue-resident macrophage populations demonstrated enhanced expression of genes causally related to fibrosis in monocytederived relative to tissue-resident cells $(13,98)$. These findings were confirmed in the same mouse model using single-cell RNASeq (99). More importantly, in patients with pulmonary fibrosis, single-cell RNA-Seq demonstrated heterogeneity in macrophage phenotypes in patients with fibrosis relative to controls, and spatial transcriptomics confirmed that "profibrotic" macrophages coexist with "normal" macrophages in the same microenvironment, supporting the hypothesis that ontogeny is an important determinant of macrophage function in human lung fibrosis $(13,100)$.

Recently, the function of resident interstitial pulmonary macrophages (which consist of two populations, peribronchial and perivascular) in tissue injury has been explored (18). In an asbestos model of lung fibrosis, these resident interstitial macrophages did not contribute to the profibrotic macrophage pool (101). In contrast, acute deletion of perivascular interstitial macrophages worsened fibrosis severity after bleomycin. These findings, however, should be treated with caution, as genetic depletion of macrophages induces their death via necrosis and may induce tissue damage on its own (21). Single-cell RNA-Seq data may identify specific markers that can be used to more precisely target distinct macrophage populations to dissect their contributions to tissue injury and repair $(76,102-105)$.

Brain. Microglia are a stable population of tissue-resident macrophages in the central nervous system. While the blood-brain barrier limits the recruitment of monocytes, they can be recruited in response to some forms of brain or spinal cord injury and differentiate into microglia (106). As in other tissues, these monocytederived macrophages appear to worsen acute injury. For example, during experimentally induced autoimmune encephalomyelitis, monocyte-derived macrophages are active drivers of CNS inflammation and are associated with disease severity $(107,108)$. However, during the resolution phase of spinal cord injury, both tissueresident and monocyte-derived microglia remove dead cells and debris, and contribute to injury resolution by releasing antiinflammatory cytokines, such as IL-10 $(109,110)$. Consistent with these findings, a growing body of work highlights the importance of scavenger receptors in microglia in slowing the progression of age-related dementias, for example those induced by the loss of apolipoprotein E (APOE) (89).

Atherosclerosis. During the development of atherosclerotic plaques, monocytes are recruited to the subendothelial space, where they differentiate into macrophages that ingest accumulated normal and modified lipoproteins to become cholesterol-laden foam cells. The balance of macrophages in the plaque is dynamic in that both macrophage numbers and the inflammatory phenotype in the plaque influence plaque fate $(111,112)$. Depletion of monocytes from the circulation using clodronate-loaded liposomes reduced plaque formation in rabbits (113), and the loss of netrin-1, which promotes monocyte/macrophage retention in the plaque, promoted the emigration of macrophages from plaques and reduced plaque severity (114). Adenoviral rescue of APOE in Apoe $e^{-/}$mice reduced monocyte recruitment and enhanced macrophage apoptosis during plaque resolution (115). Nevertheless, there is some evidence that plaque monocytes and macrophages play distinct roles in atherosclerosis pathogenesis. For example, the loss of Nr4a1 and Klf4, which are factors associated with macrophage differentiation, accelerated atherosclerosis in $A p o e^{-/-}$mice (116-119).

Gastrointestinal tract. Unlike tissue-resident macrophages in many other organs, many macrophage populations in the gut are constantly replenished by classical Ly6 $\mathrm{C}^{\text {hi }}$ circulating monocytes to maintain the intestinal macrophage pool $(14,120)$. Loss of these macrophages rapidly results in impaired gut mucosal integrity and the development of inflammation. More recently, investigators have used transcriptional profiling and lineage tracing to identify longlived self-maintaining gut macrophages in the submucosa that contribute to the maintenance of the integrity of the submucosal vasculature $(15,16)$. Both tissue-resident and recruited macrophages in the gut play key roles in the development of inflammation and the clearance of invading pathogens (121-127). These macrophages take on a less inflammatory and more reparative phenotype upon efferocytosis of apoptotic epithelial cells, but their importance in physiologic regeneration of the intestine is incompletely understood.

Liver. The liver is uniquely able to regenerate after injury (128). Several groups have shown that both monocyte-derived and tissue-resident macrophages expand during toxin-induced liver injury. Selective depletion of monocyte and tissue-resident macrophages at different times over the course of injury and recovery, and selective depletion of specific macrophage populations, variably altered the severity of liver injury and the time to resolution (129-133). Liver macrophages have also been reported to impact hepatic progenitor cell function, perhaps through macrophagederived TWEAK and Wnt3a (134-138). 
Skeletal muscle. The role of macrophages in skeletal muscle injury and repair has been primarily studied in models of acute tissue injury induced by mechanical trauma, thermal- or toxininduced injury, ischemia, and denervation. In all of these examples, recovery from injury is impaired in monocytopenic CCR2 ${ }^{-1-}$ mice, suggesting a role for monocytes and/or monocyte-derived cells in these processes (139-141). Fate mapping of recruited cells after cardiotoxin-induced injury showed that monocyte-derived macrophages progressively differentiate to promote satellite cell proliferation (142). However, the precise mechanisms by which macrophages contribute to the resolution of injury are unclear $(143,144)$. The role of resident or recruited macrophage populations in muscle loss during age-related sarcopenia, systemic inflammatory conditions, and immobility is less well studied.

\section{Further research and therapeutic potentials}

This overview raises several important questions with respect to the role of macrophages in tissue injury and repair (Figure 3). First, what are the factors/mechanisms in specific tissue microenvironments that drive macrophage differentiation/transformation into a reparative phenotype? Specifically, do these factors overlap with tissue-specific signals that drive macrophage differentiation, or do they originate from unique cells or molecules expressed during injury? Second, is the transformation of macrophages from an inflammatory phenotype to a resolution phenotype driven by their ontogeny? Specifically, do individual cells change their phenotype over the course of injury or resolution, or is there a continuous recruitment of monocyte-derived macrophages over the course of injury and resolution? Third, what is the role of less abundant tissueresident macrophage populations, such as perivascular, interstitial, or nerve-associated macrophages, in tissue homeostasis, injury, and resolution? Fourth, do different pathogens or toxins drive distinct repair phenotypes in tissue macrophages? Fifth, how do macrophages communicate with resident cell populations to promote repair? Specifically, do these mechanisms involve the restoration of homeostatic functions of tissue-resident macrophages or the active secretion of pro-repair molecules? Sixth, do epigenetic changes in resident or recruited macrophages or changes in macrophage ontogeny confer immunologic memory that might modify immunologic responses to repeated challenges or during aging? Addressing these questions will require a combination of genetic fate mapping, time series examination of macrophage epigenetic and transcriptional heterogeneity over the course of injury, high-resolution spatial transcriptomics and proteomics to identify interactions between macrophages and reparative cell populations, and confirmation in human samples and genetic knockouts. Fortunately, emerging technologies provide tractable solutions to these problems in both homeostatic and disease contexts (101).

Macrophages are attractive targets for therapy as their phenotypes differ across tissues and they can be replaced after deletion by monocyte-derived macrophages. As a result, macrophagetargeted therapies are predicted to have fewer off-target effects and be more reversible than those targeting other tissue-resident populations or circulating monocytes. Potential therapies could inhibit damaging signals originating from monocyte-derived macrophages early during injury or mimic the pro-repair functions of macrophages. Indeed, the cellular targets of several existing drugs are unknown and might include macrophages. More distinctly, the unique capacity of macrophages for phagocytosis simplifies the development of targeted delivery or deletion strategies. For example, bisphosphonates attach to hydroxyapatite binding sites on bony surfaces, where they are taken up by osteoclasts (a resident macrophage population in the bone), inducing their dysfunction or apoptosis. While this strategy is generally safe, the development of jaw osteonecrosis after dental procedures associated with bisphosphonates hints at their role in tissue repair. Macrophage-specific delivery might be enhanced through nanomaterials that are avidly phagocytosed by tissue macrophages (145). This property is already being exploited for delivery of therapy to tumor-associated macrophages (146).

\section{Acknowledgments}

SW is supported by MSD Life Science Foundation, Public Interest Incorporated Foundation; and a David W. Cugell and Christina Enroth-Cugell Fellowship. AVM is supported by NIH grants HL135124 and AI135964, Department of Defense grant PR141319, and NIH/ National Institute of Allergy and Infectious Diseases (NIAID) grant AI135964. GRSB is supported by NIH grants ES013995, HL071643, and AG049665, Veterans Administration grant BX000201, Department of Defense grant PR141319, and NIH/NIAID grant AI135964.

Address correspondence to: G.R. Scott Budinger, Northwestern University, Feinberg School of Medicine, Division of Pulmonary and Critical Care Medicine, 240 East Huron Street, Room M324, Chicago, Illinois 60611, USA. Phone: 312.503.2548; Email: s-buding@northwestern.edu.
1. Wynn TA, Chawla A, Pollard JW. Macrophage biology in development, homeostasis and disease. Nature. 2013;496(7446):445-455.

2. Lavin Y, et al. Tissue-resident macrophage enhancer landscapes are shaped by the local microenvironment. Cell. 2014;159(6):1312-1326.

3. van Furth R, Cohn ZA. The origin and kinetics of mononuclear phagocytes. J Exp Med. 1968;128(3):415-435.

4. Ginhoux F, et al. Fate mapping analysis reveals that adult microglia derive from primitive macrophages. Science. 2010;330(6005):841-845.

5 . Yona $\mathrm{S}$, et al. Fate mapping reveals origins and dynamics of monocytes and tissue macrophages under homeostasis. Immunity. 2013;38(1):79-91.

6. Hashimoto D, et al. Tissue-resident macrophages self-maintain locally throughout adult life with minimal contribution from circulating monocytes. Immunity. 2013;38(4):792-804.

7. Epelman S, et al. Embryonic and adult-derived resident cardiac macrophages are maintained through distinct mechanisms at steady state and during inflammation. Immunity. 2014;40(1):91-104.

8. Guilliams M, et al. Alveolar macrophages develop from fetal monocytes that differentiate into longlived cells in the first week of life via GM-CSF. J Exp Med. 2013;210(10):1977-1992.

9. Jakubzick C, et al. Minimal differentiation of classical monocytes as they survey steady-state tissues and transport antigen to lymph nodes. Immunity. 2013;39(3):599-610.

10. Schulz C, et al. A lineage of myeloid cells independent of Myb and hematopoietic stem cells. Science. 2012;336(6077):86-90.

11. Li Q, Barres BA. Microglia and macrophages in brain homeostasis and disease. Nat Rev Immunol. 2018;18(4):225-242.

12. Kaplan DH. Ontogeny and function of murine epidermal Langerhans cells. Nat Immunol. 2017;18(10):1068-1075.

13. Misharin AV, et al. Monocyte-derived alveolar macrophages drive lung fibrosis and per- 
sist in the lung over the life span. JExp Med. 2017;214(8):2387-2404.

14. Bain CC, Schridde A. Origin, differentiation, and function of intestinal macrophages. Front Immunol. 2018;9:2733.

15. De Schepper S, et al. Self-maintaining gut macrophages are essential for intestinal homeostasis. Cell. 2018;175(2):400-415.e13.

16. Shaw TN, et al. Tissue-resident macrophages in the intestine are long lived and defined by Tim- 4 and CD 4 expression. JExp Med. 2018;215(6):1507-1518.

17. Tabula Muris Consortium, et al. Single-cell transcriptomics of 20 mouse organs creates a Tabula Muris. Nature. 2018;562(7727):367-372.

18. Gibbings SL, et al. Three unique interstitial macrophages in the murine lung at steady state. $\mathrm{Am} \mathrm{J}$ Respir Cell Mol Biol. 2017;57(1):66-76.

19. Rahman K, et al. Inflammatory Ly6Chi monocytes and their conversion to M2 macrophages drive atherosclerosis regression. J Clin Invest. 2017;127(8):2904-2915.

20. Dick SA, et al. Self-renewing resident cardiac macrophages limit adverse remodeling following myocardial infarction. Nat Immunol. 2019;20(1):29-39.

21. Chakarov S, et al. Two distinct interstitial macrophage populations coexist across tissues in specific subtissular niches. Science. 2019;363(6432):eaau0964.

22. Ginhoux F, Guilliams M. Tissue-resident macrophage ontogeny and homeostasis. Immunity. 2016;44(3):439-449.

23. Gautier EL, et al. Gene-expression profiles and transcriptional regulatory pathways that underlie the identity and diversity of mouse tissue macrophages. Nat Immunol. 2012;13(11):1118-1128.

24. Gosselin D, et al. Environment drives selection and function of enhancers controlling tissue-specific macrophage identities. Cell. 2014;159(6):1327-1340.

25. Guth AM, Janssen WJ, Bosio CM, Crouch EC, Henson PM, Dow SW. Lung environment determines unique phenotype of alveolar macrophages. Am J Physiol Lung Cell Mol Physiol. 2009;296(6):L936-L946.

26. van de Laar L, et al. Yolk sac macrophages, fetal liver, and adult monocytes can colonize an empty niche and develop into functional tissue-resident macrophages. Immunity. 2016;44(4):755-768.

27. Laskin DL, Sunil VR, Gardner CR, Laskin JD. Macrophages and tissue injury: agents of defense or destruction? Annu Rev Pharmacol Toxicol. 2011;51:267-288.

28. Gordon S, Plüddemann A, Martinez Estrada F. Macrophage heterogeneity in tissues: phenotypic diversity and functions. Immunol Rev. 2014;262(1):36-55.

29. Mould KJ, et al. Cell origin dictates programming of resident versus recruited macrophages during acute lung injury. Am J Respir Cell Mol Biol. 2017;57(3):294-306.

30. Xiong $\mathrm{H}$, et al. Distinct contributions of neutrophils and CCR2 ${ }^{+}$monocytes to pulmonary clearance of different Klebsiella pneumoniae strains. Infect Immun. 2015;83(9):3418-3427.

31. Carr KD, Sieve AN, Indramohan M, Break TJ, Lee S, Berg RE. Specific depletion reveals a novel role for neutrophil-mediated protection in the liver during Listeria monocytogenes infection. Eur J Immunol. 2011;41(9):2666-2676.

32. Coates BM, et al. Inflammatory monocytes drive influenza a virus-mediated lung injury in juvenile mice. JImmunol. 2018;200(7):2391-2404.

33. Mould KJ, Jackson ND, Henson PM, Seibold M, Janssen WJ. Single cell RNA sequencing identifies unique inflammatory airspace macrophage subsets. JCI Insight. 2019;4(5):126556.

34. Schneider C, Nobs SP, Kurrer M, Rehrauer H, Thiele C, Kopf M. Induction of the nuclear receptor PPAR- $\gamma$ by the cytokine GM-CSF is critical for the differentiation of fetal monocytes into alveolar macrophages. Nat Immunol. 2014;15(11):1026-1037.

35. Yu X, et al. The cytokine TGF- $\beta$ promotes the development and homeostasis of alveolar macrophages. Immunity. 2017;47(5):903-912.

36. Sinclair C, et al. mTOR regulates metabolic adaptation of APCs in the lung and controls the outcome of allergic inflammation. Science. 2017;357(6355):1014-1021.

37. Rosas $\mathrm{M}$, et al. The transcription factor Gata6 links tissue macrophage phenotype and proliferative renewal. Science. 2014;344(6184):645-648

38. Aziz A, Soucie E, Sarrazin S, Sieweke MH. $\mathrm{MafB} / \mathrm{c}-\mathrm{Maf}$ deficiency enables self-renewal of differentiated functional macrophages. Science. 2009;326(5954):867-871.

39. Shi J, Hua L, Harmer D, Li P, Ren G. Cre driver mice targeting macrophages. Methods Mol Biol. 2018;1784:263-275.

40. Mills CD, Kincaid K, Alt JM, Heilman MJ, Hill AM. M-1/M-2 macrophages and the Th1/Th2 paradigm. JImmunol. 2000;164(12):6166-6173.

41. Mills CD. M1 and M2 macrophages: oracles of health and disease. Crit Rev Immunol. 2012;32(6):463-488.

42. Xue J, et al. Transcriptome-based network analysis reveals a spectrum model of human macrophage activation. Immunity. 2014;40(2):274-288.

43. Avraham R, et al. Pathogen cell-to-cell variability drives heterogeneity in host immune responses. Cell. 2015;162(6):1309-1321.

44. Janssen WJ, et al. Fas determines differential fates of resident and recruited macrophages during resolution of acute lung injury. Am J Respir Crit Care Med. 2011;184(5):547-560.

45. Reyfman PA, et al. Single-cell transcriptomic analysis of human lung provides insights into the pathobiology of pulmonary fibrosis [published online ahead of print December 15, 2018]. Am J Respir Crit Care Med.https://doi.org/10.1164/rccm.20171224100C.

46. Guilliams M, Mildner A, Yona S. Developmental and functional heterogeneity of monocytes. Immunity. 2018;49(4):595-613.

47. Nahrendorf M, Swirski FK. Abandoning M1/M2 for a network model of macrophage function. Circ Res. 2016;119(3):414-417.

48. Vannella KM, Wynn TA. Mechanisms of organ injury and repair by macrophages. Annu Rev Physiol. 2017;79:593-617.

49. Tay TL, et al. A new fate mapping system reveals context-dependent random or clonal expansion of microglia. Nat Neurosci. 2017;20(6):793-803.

50. Hanayama R, et al. Autoimmune dis- ease and impaired uptake of apoptotic cells in MFG-E8-deficient mice. Science. 2004;304(5674):1147-1150.

51. A-Gonzalez N, et al. Phagocytosis imprints heterogeneity in tissue-resident macrophages. J Exp Med. 2017;214(5):1281-1296.

52. Lemke G. Biology of the TAM receptors. Cold Spring Harb Perspect Biol. 2013;5(11):a009076.

53. Kourtzelis I, et al. DEL-1 promotes macrophage efferocytosis and clearance of inflammation. Nat Immunol. 2019;20(1):40-49.

54. Yanagihashi Y, Segawa K, Maeda R, Nabeshima YI, Nagata S. Mouse macrophages show different requirements for phosphatidylserine receptor Tim4 in efferocytosis. Proc Natl Acad Sci U S A. 2017;114(33):8800-8805.

55. Arpaia N, et al. A distinct function of regulatory T cells in tissue protection. Cell. 2015;162(5):1078-1089.

56. Bourdonnay E, et al. Transcellular delivery of vesicular SOCS proteins from macrophages to epithelial cells blunts inflammatory signaling. JExp Med. 2015;212(5):729-742.

57. Roy S. miRNA in macrophage development and function. Antioxid Redox Signal. 2016;25(15):795-804.

58. Basrai HS, Christie KJ, Turbic A, Bye N, Turnley AM. Suppressor of Cytokine Signaling-2 (SOCS2) regulates the microglial response and improves functional outcome after traumatic brain injury in mice. PLoS One. 2016;11(4):e0153418.

59. Fredman G, Li Y, Dalli J, Chiang N, Serhan CN. Self-limited versus delayed resolution of acute inflammation: temporal regulation of pro-resolving mediators and microRNA. Sci Rep. 2012;2:639.

60. Recchiuti A, Krishnamoorthy S, Fredman G, Chiang N, Serhan CN. MicroRNAs in resolution of acute inflammation: identification of novel resolvin D1-miRNA circuits. FASEB J. 2011;25(2):544-560.

61. Murata Y, Saito Y, Kotani T, Matozaki T. CD47-signal regulatory protein $\alpha$ signaling system and its application to cancer immunotherapy. Cancer Sci. 2018;109(8):2349-2357.

62. Manich G, Recasens M, Valente T, Almolda B, González B, Castellano B. Role of the CD200CD200R axis during homeostasis and neuroinflammation [published online ahead of print October 24, 2018]. Neuroscience. https://doi. org/10.1016/j.neuroscience.2018.10.030.

63. Van den Bossche J, Malissen B, Mantovani A, De Baetselier P, Van Ginderachter JA. Regulation and function of the E-cadherin/catenin complex in cells of the monocyte-macrophage lineage and DCs. Blood. 2012;119(7):1623-1633.

64. Zhang MZ, et al. CSF-1 signaling mediates recovery from acute kidney injury. J Clin Invest. 2012;122(12):4519-4532.

65. Morgan DJ, et al. Innate immune cell suppression and the link with secondary lung bacterial pneumonia. Front Immunol. 2018;9:2943.

66. Smigiel KS, Parks WC. Matrix metalloproteinases and leukocyte activation. Prog Mol Biol Transl Sci. 2017;147:167-195.

67. Rohani MG, et al. MMP-10 regulates collagenolytic activity of alternatively activated resident macrophages. J Invest Dermatol. 2015;135(10):2377-2384.

68. Serhan CN, Levy BD. Resolvins in inflam- 
mation: emergence of the pro-resolving superfamily of mediators. J Clin Invest. 2018;128(7):2657-2669.

69. Zhang S, et al. Efferocytosis fuels requirements of fatty acid oxidation and the electron transport chain to polarize macrophages for tissue repair. Cell Metab. 2019;29(2):443-456.e5.

70. Jha AK, et al. Network integration of parallel metabolic and transcriptional data reveals metabolic modules that regulate macrophage polarization. Immunity. 2015;42(3):419-430.

71. Morioka S, et al. Efferocytosis induces a novel SLC program to promote glucose uptake and lactate release. Nature. 2018;563(7733):714-718.

72. O'Neill LAJ, Artyomov MN. Itaconate: the poster child of metabolic reprogramming in macrophage function [published online ahead of print January 31, 2019]. Nat Rev Immunol. https://doi. org/10.1038/s41577-019-0128-5.

73. Linke $\mathrm{M}$, et al. Chronic signaling via the metabolic checkpoint kinase mTORC1 induces macrophage granuloma formation and marks sarcoidosis progression. Nat Immunol. 2017;18(3):293-302.

74. Bah A, Vergne I. Macrophage autophagy and bacterial infections. Front Immunol. 2017;8:1483.

75. Cronan MR, et al. Macrophage epithelial reprogramming underlies mycobacterial granuloma formation and promotes infection. Immunity. 2016;45(4):861-876

76. Gibbons MA, et al. Ly6Chi monocytes direct alternatively activated profibrotic macrophage regulation of lung fibrosis. Am J Respir Crit Care Med. 2011;184(5):569-581.

77. Madsen DH, et al. M2-like macrophages are responsible for collagen degradation through a mannose receptor-mediated pathway. JCell Biol. 2013;202(6):951-966.

78. Madsen DH, et al. Tumor-associated macrophages derived from circulating inflammatory monocytes degrade collagen through cellular uptake. Cell Rep. 2017;21(13):3662-3671.

79. Gibbings SL, et al. Transcriptome analysis highlights the conserved difference between embryonic and postnatal-derived alveolar macrophages. Blood. 2015;126(11):1357-1366.

80. Seeley JJ, et al. Induction of innate immune memory via microRNA targeting of chromatin remodelling factors. Nature. 2018;559(7712):114-119.

81. Yao Y, et al. Induction of autonomous memory alveolar macrophages requires $\mathrm{T}$ cell help and is critical to trained immunity. Cell. 2018;175(6):1634-1650.e17.

82. Budinger GRS, et al. The intersection of aging biology and the pathobiology of lung diseases: a joint NHLBI/NIA workshop. J Gerontol A Biol Sci Med Sci. 2017;72(11):1492-1500.

83. Linehan E, Fitzgerald DC. Ageing and the immune system: focus on macrophages. Eur $J$ Microbiol Immunol (Bp). 2015;5(1):14-24.

84. Swift ME, Kleinman HK, DiPietro LA. Impaired wound repair and delayed angiogenesis in aged mice. Lab Invest. 1999;79(12):1479-1487.

85. Swift ME, Burns AL, Gray KL, DiPietro LA. Age-related alterations in the inflammatory response to dermal injury. JInvest Dermatol. 2001;117(5):1027-1035.

86. Gosain A, DiPietro LA. Aging and wound healing.
World J Surg. 2004;28(3):321-326.

87. Danon D, Kowatch MA, Roth GS. Promotion of wound repair in old mice by local injection of macrophages. Proc Natl Acad Sci US A. 1989;86(6):2018-2020.

88. Yuan X, et al. Macrophage depletion ameliorates peripheral neuropathy in aging mice. J Neurosci. 2018;38(19):4610-4620.

89. Sarlus H, Heneka MT. Microglia in Alzheimer's disease. JClin Invest. 2017;127(9):3240-3249.

90. Camell CD, et al. Inflammasome-driven catecholamine catabolism in macrophages blunts lipolysis during ageing. Nature. 2017;550(7674):119-123.

91. Frantz S, Nahrendorf M. Cardiac macrophages and their role in ischaemic heart disease. Cardiovasc Res. 2014;102(2):240-248.

92. Hulsmans M, et al. Macrophages facilitate electrical conduction in the heart. Cell. 2017;169(3):510-522.e20.

93. Bajpai G, et al. Tissue resident CCR2 and CCR $2^{+}$cardiac macrophages differentially orchestrate monocyte recruitment and fate specification following myocardial injury. Circ Res. 2019;124(2):263-278.

94. Panizzi $\mathrm{P}$, et al. Impaired infarct healing in atherosclerotic mice with Ly-6C(hi) monocytosis. JAm Coll Cardiol. 2010;55(15):1629-1638.

95. Howangyin KY, et al. Myeloid-epithelial-reproductive receptor tyrosine kinase and milk fat globule epidermal growth factor 8 coordinately improve remodeling after myocardial infarction via local delivery of vascular endothelial growth factor. Circulation. 2016;133(9):826-839.

96. Happle C, et al. Pulmonary transplantation of macrophage progenitors as effective and long-lasting therapy for hereditary pulmonary alveolar proteinosis. Sci Transl Med. 2014;6(250):250ra113.

97. Suzuki T, et al. Pulmonary macrophage transplantation therapy. Nature. 2014;514(7523):450-454.

98. Moore BB, et al. Protection from pulmonary fibrosis in the absence of CCR2 signaling. JImmunol. 2001;167(8):4368-4377.

99. Aran D, et al. Reference-based analysis of lung single-cell sequencing reveals a transitional profibrotic macrophage. Nat Immunol. 2019;20(2):163-172.

100.McCubbrey AL, et al. Deletion of c-FLIP from $\mathrm{CD} 11 \mathrm{~b}^{\mathrm{hi}}$ macrophages prevents development of bleomycin-induced lung fibrosis. Am J Respir Cell Mol Biol. 2018;58(1):66-78.

101.Joshi N, et al. Single-cell RNA-seq reveals spatially restricted multicellular fibrotic niches during lung fibrosis. bioRxiv Website. Published March 6, 2019. Accessed April 14, 2019. https://doi. org $/ 10.1101 / 569855$.

102. Machiels B, et al. A gammaherpesvirus provides protection against allergic asthma by inducing the replacement of resident alveolar macrophages with regulatory monocytes. Nat Immunol. 2017;18(12):1310-1320.

103. Ikeda N, et al. Emergence of immunoregulatory Ym1. Sci Immunol. 2018;3(28):eaat0207.

104.Allard B, Panariti A, Martin JG. Alveolar macrophages in the resolution of inflammation, tissue repair, and tolerance to infection. Front Immunol. 2018;9:1777.
105. Guilliams M, Scott CL. Does niche competition determine the origin of tissue-resident macrophages? Nat Rev Immunol. 2017;17(7):451-460.

106.Jung S, Schwartz M. Non-identical twins microglia and monocyte-derived macrophages in acute injury and autoimmune inflammation. Front Immunol. 2012;3:89.

107. Mildner A, et al. CCR2 $2^{+} \mathrm{Ly}-6 \mathrm{C}^{\text {hi }}$ monocytes are crucial for the effector phase of autoimmunity in the central nervous system. Brain. 2009;132(pt 9):2487-2500.

108.Ajami B, Bennett JL, Krieger C, McNagny KM, Rossi FM. Infiltrating monocytes trigger EAE progression, but do not contribute to the resident microglia pool. Nat Neurosci. 2011;14(9):1142-1149.

109. Gordon S, Taylor PR. Monocyte and macrophage heterogeneity. Nat Rev Immunol. 2005;5(12):953-964.

110.Shechter R, et al. Infiltrating blood-derived macrophages are vital cells playing an anti-inflammatory role in recovery from spinal cord injury in mice. PLoS Med. 2009;6(7):e1000113.

111. Woollard KJ, Geissmann F. Monocytes in atherosclerosis: subsets and functions. Nat Rev Cardiol. 2010;7(2):77-86.

112. Moore KJ, Sheedy FJ, Fisher EA. Macrophages in atherosclerosis: a dynamic balance. Nat Rev Immunol. 2013;13(10):709-721.

113. Ylitalo R, Oksala O, Ylä-Herttuala S, Ylitalo P. Effects of clodronate (dichloromethylene bisphosphonate) on the development of experimental atherosclerosis in rabbits. J Lab Clin Med. 1994;123(5):769-776.

114. van Gils JM, et al. The neuroimmune guidance cue netrin-1 promotes atherosclerosis by inhibiting the emigration of macrophages from plaques. Nat Immunol. 2012;13(2):136-143.

115. Ravichandran KS. Beginnings of a good apoptotic meal: the find-me and eat-me signaling pathways. Immunity. 2011;35(4):445-455.

116. Liao X, et al. Krüppel-like factor 4 regulates macrophage polarization. J Clin Invest. 2011;121(7):2736-2749.

117. Hamers AA, et al. Bone marrow-specific deficiency of nuclear receptor Nur77 enhances atherosclerosis. Circ Res. 2012;110(3):428-438.

118. Hanna RN, et al. NR4A1 (Nur77) deletion polarizes macrophages toward an inflammatory phenotype and increases atherosclerosis. Circ Res. 2012;110(3):416-427.

119. Sharma N, et al. Myeloid Krüppel-like factor 4 deficiency augments atherogenesis in $\mathrm{ApoE}^{-/-}$ mice - brief report. Arterioscler Thromb Vasc Biol. 2012;32(12):2836-2838.

120. Bain CC, et al. Constant replenishment from circulating monocytes maintains the macrophage pool in the intestine of adult mice. Nat Immunol. 2014;15(10):929-937.

121. Bain CC, et al. Resident and pro-inflammatory macrophages in the colon represent alternative context-dependent fates of the same $\mathrm{Ly}^{6} \mathrm{C}^{\text {hi }}$ monocyte precursors. Mucosal Immunol. 2013;6(3):498-510.

122. Weber B, Saurer L, Schenk M, Dickgreber N, Mueller C. CX3CR1 defines functionally distinct intestinal mononuclear phagocyte subsets which maintain their respective functions during homeostatic and inflammatory conditions. Eur J 
Immunol. 2011;41(3):773-779.

123. Zigmond $\mathrm{E}$, et al. Ly6C $\mathrm{C}^{\text {hi }}$ monocytes in the inflamed colon give rise to proinflammatory effector cells and migratory antigen-presenting cells. Immunity. 2012;37(6):1076-1090.

124. Dunay IR, et al. Gr1(+) inflammatory monocytes are required for mucosal resistance to the pathogen Toxoplasma gondii. Immunity. 2008;29(2):306-317.

125. Kim YG, et al. The Nod 2 sensor promotes intestinal pathogen eradication via the chemokine CCL2-dependent recruitment of inflammatory monocytes. Immunity. 2011;34(5):769-780.

126. Grainger JR, et al. Inflammatory monocytes regulate pathologic responses to commensals during acute gastrointestinal infection. Nat Med. 2013;19(6):713-721.

127. Farro G, et al. CCR2-dependent monocyte-derived macrophages resolve inflammation and restore gut motility in postoperative ileus. Gut. 2017;66(12):2098-2109.

128.Duncan AW, Dorrell C, Grompe M. Stem cells and liver regeneration. Gastroenterology. 2009;137(2):466-481.

129. Duffield JS, et al. Selective depletion of macrophages reveals distinct, opposing roles during liver injury and repair. J Clin Invest. 2005;115(1):56-65.

130. Ramachandran P, et al. Differential Ly-6C expression identifies the recruited macrophage phenotype, which orchestrates the regression of murine liver fibrosis. Proc Natl Acad Sci U S A.
2012;109(46):E3186-E3195.

131. Dal-Secco D, et al. A dynamic spectrum of monocytes arising from the in situ reprogramming of CCR2 ${ }^{+}$monocytes at a site of sterile injury. J Exp Med. 2015;212(4):447-456.

132. Stutchfield BM, et al. CSF1 restores innate immunity after liver injury in mice and serum levels indicate outcomes of patients with acute liver failure. Gastroenterology. 2015;149(7):1896-1909.e14.

133. Antoniades CG, et al. Source and characterization of hepatic macrophages in acetaminophen-induced acute liver failure in humans. Hepatology. 2012;56(2):735-746.

134.Xiang S, et al. Oval cell response is attenuated by depletion of liver resident macrophages in the 2-AAF/partial hepatectomy rat. PLoS One. 2012;7(4):e35180.

135. Thomas JA, et al. Macrophage therapy for murine liver fibrosis recruits host effector cells improving fibrosis, regeneration, and function. Hepatology. 2011;53(6):2003-2015.

136. Jakubowski A, et al. TWEAK induces liver progenitor cell proliferation. J Clin Invest. 2005;115(9):2330-2340.

137. Karaca G, et al. TWEAK/Fn14 signaling is required for liver regeneration after partial hepatectomy in mice. PLoS One. 2014;9(1):e83987.

138. Boulter L, et al. Macrophage-derived Wnt opposes Notch signaling to specify hepatic progenitor cell fate in chronic liver disease. Nat Med. 2012;18(4):572-579.

139. Yang W, Hu P. Skeletal muscle regeneration is modulated by inflammation. JOrthop Translat. 2018;13:25-32.

140. Arnold L, et al. Inflammatory monocytes recruited after skeletal muscle injury switch into antiinflammatory macrophages to support myogenesis. JExp Med. 2007;204(5):1057-1069.

141. Sun D, et al. Bone marrow-derived cell regulation of skeletal muscle regeneration. FASEB J. 2009;23(2):382-395.

142. Saclier M, Cuvellier S, Magnan M, Mounier R, Chazaud B. Monocyte/macrophage interactions with myogenic precursor cells during skeletal muscle regeneration. FEBS J. 2013;280(17):4118-4130.

143. Wang H, Melton DW, Porter L, Sarwar ZU, McManus LM, Shireman PK. Altered macrophage phenotype transition impairs skeletal muscle regeneration. Am J Pathol. 2014;184(4):1167-1184.

144. Varga T, et al. Highly dynamic transcriptional signature of distinct macrophage subsets during sterile inflammation, resolution, and tissue repair. J Immunol. 2016;196(11):4771-4782.

145. Duch MC, et al. Minimizing oxidation and stable nanoscale dispersion improves the biocompatibility of graphene in the lung. Nano Lett. 2011;11(12):5201-5207.

146. Ovais M, Guo M, Chen C. Tailoring nanomaterials for targeting tumor-associated macrophages [published online ahead of print March 18, 2019]. Adv Mater. https://doi.org/10.1002/ adma.201808303. 